\title{
Solitary Osteochondroma of the Skull Base: A Case Report and Literature Review
}

\author{
Hiroki Hongo $^{1}$ Soichi Oya ${ }^{1}$ Atsushi Abe ${ }^{2}$ Toru Matsui $^{1}$ \\ ${ }^{1}$ Department of Neurosurgery, Saitama Medical Center, Saitama \\ Medical University, Saitama, Japan \\ 2 Department of Radiology, Saitama Medical Center, Saitama Medical \\ University, Saitama, Japan \\ Address for correspondence Soichi Oya, MD, PhD, Department of \\ Neurosurgery, Saitama Medical Center, Kamoda 1981, Kawagoe, \\ Saitama, 350-8550, Japan (e-mail: sooya-tky@umin.ac.jp).
}

J Neurol Surg Rep 2015;76:e13-e17.

\begin{abstract}
Keywords

- abducens nerve palsy

- bone tumor

- osteochondroma

- posterior clinoid

- skull base

We report a case of an osteochondroma in the posterior clinoid process that occurred in a 43-year-old man with trochlear nerve palsy. Although the potential preoperative diagnoses based on computed tomography and magnetic resonance imaging included other intracranial tumors such as calcified meningioma, thallium-201 single-photon emission computed tomography effectively differentiated osteochondroma from those possibilities. Via an orbitozygomatic approach, a subtotal resection was achieved with a good relief of symptoms. Twenty-two cases of solitary osteochondromas in the skull base have been reported that have demonstrated little risk of recurrence or malignant transformation. However, surgery for skull base osteochondromas does carry a significant risk with a reported mortality $>10 \%$. Although some previous reports advocate complete resection as the only curative method for skull base osteochondromas, the risks of total resection should be weighed against the chance for recurrence; our review of the literature demonstrated a relatively high mortality and an extremely low incidence of recurrence.
\end{abstract}

\section{Introduction}

An osteochondroma is a benign tumor composed of mature hyaline cartilage with focal ossification. ${ }^{1}$ It constitutes 10 to $15 \%$ of all bone tumors and 20 to $50 \%$ of benign bone tumors ${ }^{2}$ and is frequently found at the epiphysis of long bones such as the distal femur, proximal tibia, and proximal humerus. However, intracranial osteochondromas are relatively rare, and the literature consists only of sporadic case reports. ${ }^{1,3-23}$ We report a rare case of an intracranial osteochondroma of the posterior clinoid process that caused right abducens nerve palsy along with a review of the literature.

\section{Case Report}

A 43-year-old man with no past medical history developed diplopia 3 years ago. He consulted an ophthalmologist, who found no ophthalmologic disease. Because the symptom gradually subsided over several months, he was treated conservatively. Approximately 18 months after the onset, his diplopia recurred; this time, it did not subside and gradually deteriorated. He developed a continuous medial excursion of the right eye. He visited a general hospital, where he was diagnosed with right abducens nerve palsy. Magnetic resonance (MR) images obtained at the hospital revealed an intracranial mass in the right skull base region, and he was referred to our hospital. A detailed neurologic examination on the initial visit revealed complete abducens nerve palsy with no other cranial nerve deficit. He had no muscle weakness or sensory disturbance. A noncontrast computed tomography scan demonstrated a $3.0 \times 2.5 \times 2.5 \mathrm{~cm}$ well-circumscribed extra-axial mass lesion in the right posterior clinoid that contained numerous irregular calcified spots ( - Fig. 1A, B). The lesion was located in the right posterior clinoid process of received

March 20, 2014 accepted after revision June 30,2014

published online

March 2, 2015
DOI http://dx.doi.org/

10.1055/s-0034-1387189. ISSN 2193-6366. (c) 2015 Georg Thieme Verlag KG
Stuttgart · New York

License terms

((1) $\circledast$ 

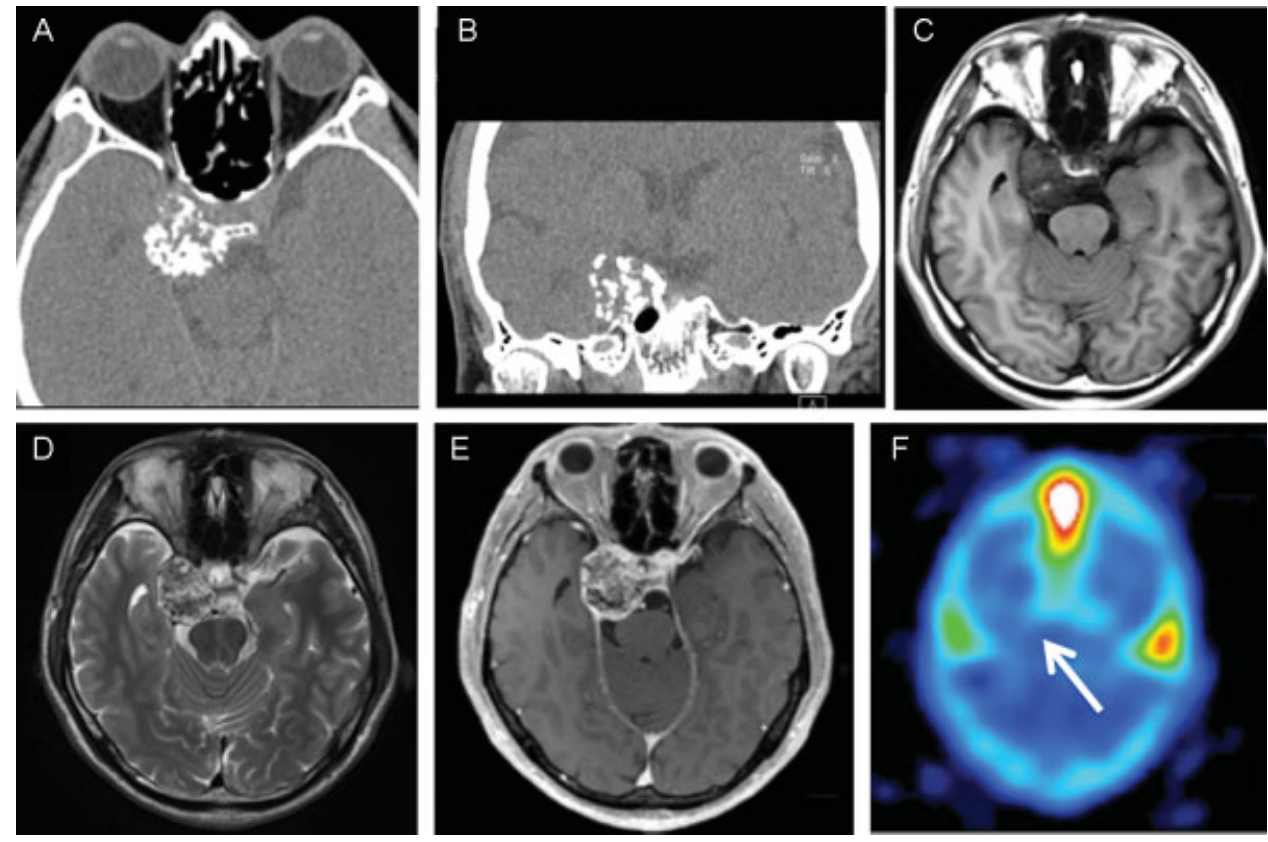

Fig. 1 (A, B) Preoperative computed tomograms showing a mass with atypical patterns of calcification in the skull base. (C) Axial T1-weighted magnetic resonance (MR) imaging demonstrating a mass in the right posterior clinoid with low intensity. (D) Axial T2-weighted MR imaging depicting a mass with heterogeneous intensity. (E) Axial T1-weighted MR imaging with gadolinium showing a mass with heterogeneous enhancement. (F) The mass demonstrated an extremely low uptake on thallium-201 single-photon emission computed tomography.

the sphenoid bone and caused the destruction of the petrous apex of the temporal bone. MR imaging showed that the lesion had heterogeneous signals of intermediate to low intensity on the T1-weighted images ( - Fig. 1C) and of low to high intensity on the T2-weighted images ( - Fig. 1D). The T1-weighted images with gadolinium enhancement showed strong enhancement of the noncalcified areas (-Fig. 1E). The dural tail sign was not apparent, and there was no perifocal edema. Cerebral angiography showed no tumor stain or arterial stenosis. Thallium-201 single-photon emission computed tomography (SPECT) revealed an extremely low uptake for meningothelial tumors. The preoperative differential diagnosis included meningioma, cavernous hemangioma with intralesional calcification, miscellaneous osteomatous lesions, sarcoma, fibrous dysplasia, and metastasis.

To relieve the abducens nerve palsy and make a definitive diagnosis, a surgical resection via right orbitozygomatic craniotomy was performed while monitoring the extraocular muscles. The right optic nerve, right internal carotid artery, and medial right incisura of the tentorium were exposed by opening the proximal sylvian fissure. The surface of tentorium bulged upward significantly (-Fig. 2 A). The tentorium was cut, and the tumor was readily identified; it mildly adhered to the oculomotor nerve. Because the tumor was composed of white brittle tissue with multiple small calcified masses, we removed it piecemeal (-Fig. 2B). There was a substantial amount of bleeding from the cavernous sinus, but it was controlled with a small piece of Gelfoam. Intraoperative frozen sections were unavailable because the specimen was essentially calcified; nevertheless, we were convinced the lesion was benign rather than malignant from our intraoperative findings. Therefore, we intentionally left the thin tumor capsule to prevent the leakage of blood from the cavernous sinus to the prepontine cistern. Although the trochlear and abducens nerves were not found inside the tumor and the oculomotor nerve was preserved anatomically, we were unable to detect extraocular muscle movement during the intraoperative monitoring probably because of compression to the nerves by the surgical manipulation.

Postoperative MR images confirmed near total resection (-Fig. 2C). The pathologic findings demonstrated that the mass was macroscopically composed of bone and white substantial tissue like cartilage. On microscopic findings, there was bony tissue including bone marrow, trabecular bone, and fragments of hyaline cartilage comprising clusters of benign mature and degenerating chondrocytes with foci of irregular calcification and ossification (-Fig. 2D). The bone marrow was occupied by adipose tissue and fibrosis. These findings were consistent with the characteristics of osteochondroma.

Right oculomotor nerve palsy was newly observed after surgery, and the palsy completely resolved in 4 months. Although the abducens nerve palsy had persisted for $>18$ months preoperatively, there was evidence of an almost full recovery at the 12-month follow-up after surgery.

\section{Discussion}

Osteochondromas are benign tumors composed of mature hyaline cartilage with focal ossification. ${ }^{1}$ It is the most common tumor of the bone arising in any part of the body, but it is rare in the skull. ${ }^{17}$ We conducted a comprehensive literature search using the online database and identified 22 cases of osteochondromas of the skull including our case (-Table 1). Nineteen of the 22 patients (86.4\%) were men. Eleven cases (50.0\%) arose in the skull base, whereas the 

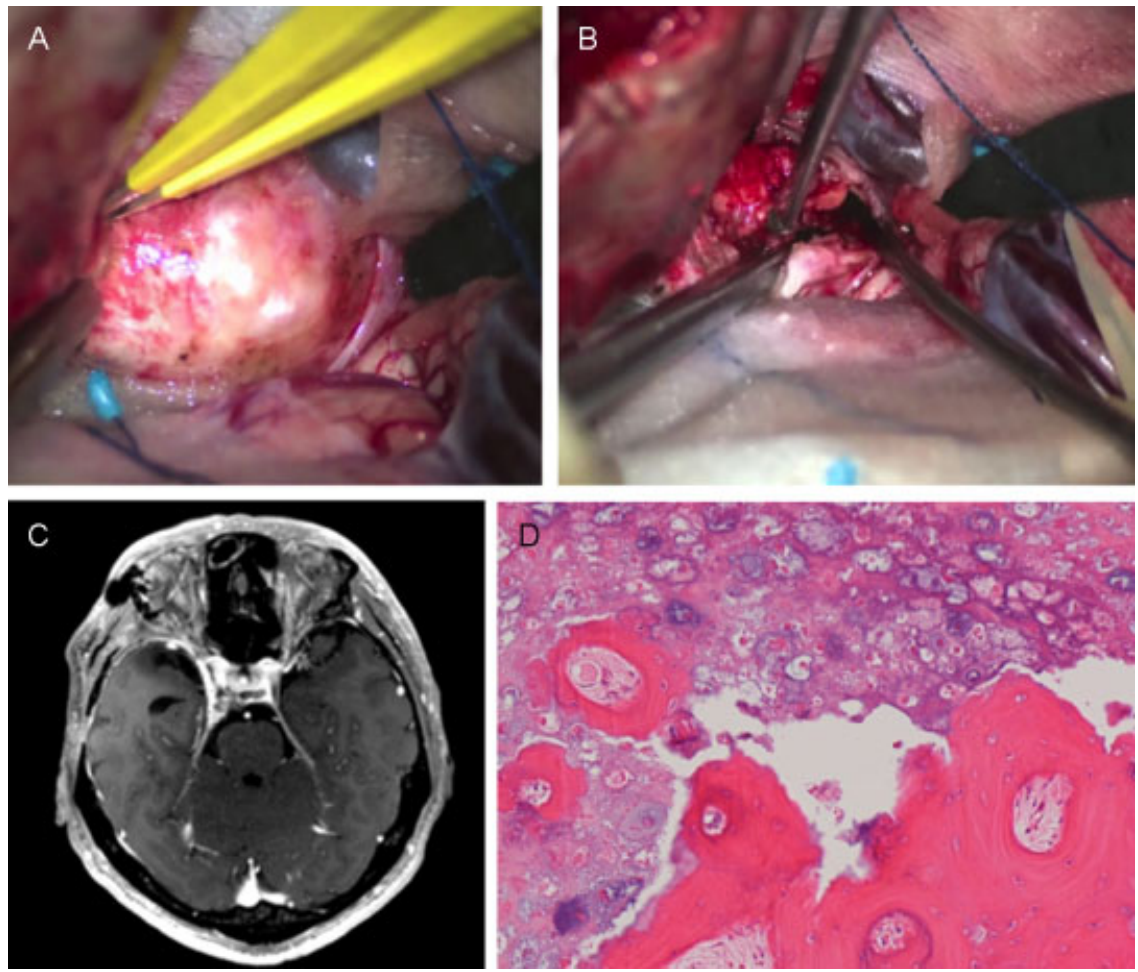

Fig. 2 (A) Intraoperative photograph showing the tumor covered by the tentorium. (B) The tumor was composed of brittle small calcified particles and hemorrhagic soft tumor components. (C) Postoperative T1-weighted magnetic resonance image with gadolinium demonstrating subtotal resection of the tumor. (D) Photomicrograph showing that the tumor consisted of bony tissue including bone marrow, trabecular bone, and benign hyaline cartilage. Original magnification: $\times 100$.

others were found in the convexity and the falx. Three cases including ours occurred in the posterior clinoid. As shown in -Table 1, 80\% of the osteochondromas in the skull base presented with focal cranial nerve deficits depending on the regions affected by the tumor, whereas non-skull base osteochondromas frequently caused epilepsy and headache as an initial symptom. Apart from visual disturbances, abducens nerve palsy was the most common cranial nerve deficit among skull base osteochondromas. This is probably related to the origin of the tumor cells, the cartilage rests in the synchondrosis. ${ }^{1}$ As shown in - Table 1, skull base osteochondromas frequently arise in the parasellar region including the posterior clinoid and the middle cranial fossa, which is close to the confluence where the sphenopetrosal, spheno-occipital, and petro-occipital synchondrosis meet. ${ }^{12,19}$ We therefore speculate that the abducens nerve palsy is most frequently observed in skull base osteochondromas because the nerve runs closer to these synchondroses than other cranial nerves.

Some previous reports mentioned that osteochondroma can mimic meningioma. 1,12,15,22 Osteochondromas are usually heterogeneously enhanced on T1-weighted images with gadolinium, ${ }^{12,14,18,21}$ indicating that contrast-enhanced MR images are not useful in completely discerning osteochondroma from meningiomas. Angiography has a diagnostic value because osteochondroma appears as avascular masses. ${ }^{1,8-11,16,17,20,23-25}$ In our experience, the findings of an extremely low uptake on thallium-201 SPECT were useful in differentiating osteochondroma from other intracranial tumors such as meningioma.

As for treatment, complete surgical removal is considered the only curative treatment for extracranial osteochondromas. ${ }^{2,22}$ Venkata et al mentioned that skull base osteochondromas may recur from incomplete resection. ${ }^{22}$ However, although gross total resection was achieved in only 11 of 23 cases $(47.8 \%)$, only two recurrent cases (8.7\%) have been reported in the literature. ${ }^{23,26}$ In addition, in our literature search, we did not find any reports of malignant transformation of solitary intracranial osteochondroma except for one case reported in $1935 .^{26}$ The incidence of malignant transformation from a solitary osteochondroma in other parts of the body was $<1 \%$, whereas that from multiple osteochondromas was reported as 0.5 to $5 \% .{ }^{26}$ Based on these facts, skull base osteochondromas are basically benign lesions, especially for solitary lesions; the risks of radical resection should be carefully weighed against the future risk of recurrence or malignant transformation. When a small asymptomatic bony lesion showed similar radiologic characteristics, a conservative management strategy could be used. Skull base osteochondromas are often located close to critical structures such as the internal carotid artery, the cavernous sinus, and the cranial nerves. Given that the mortality after surgery occurred in three patients (13.6\%) in the literature, ${ }^{4,13,19}$ subtotal or even partial resections can be an important option to relieve the symptoms and achieve good long-term tumor control. 


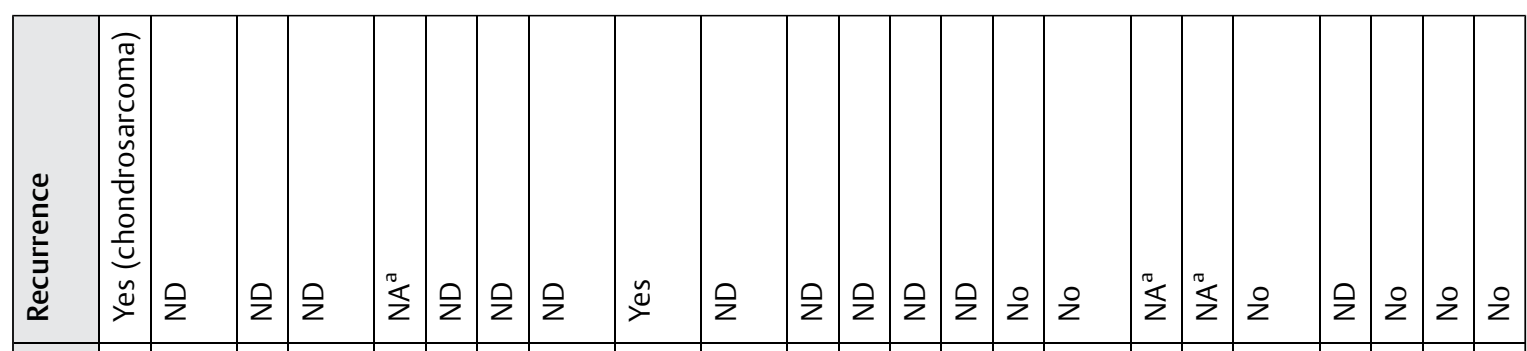

\section{产产}

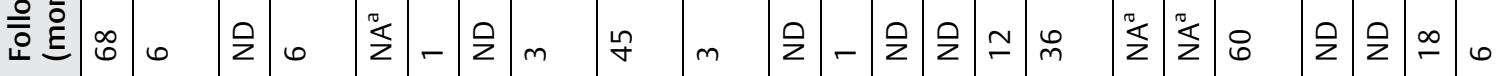

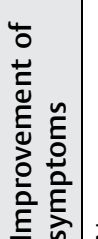

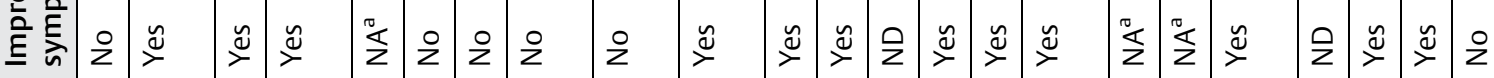

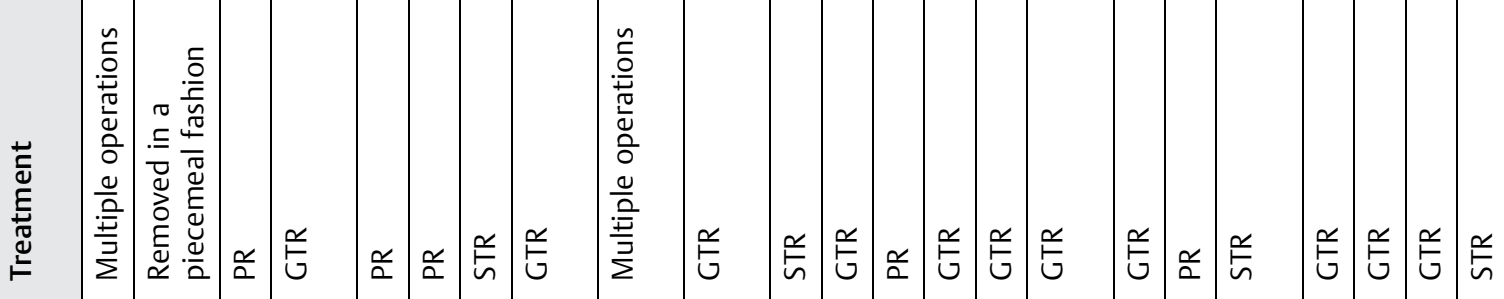

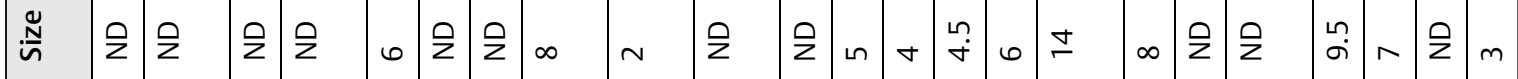

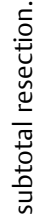

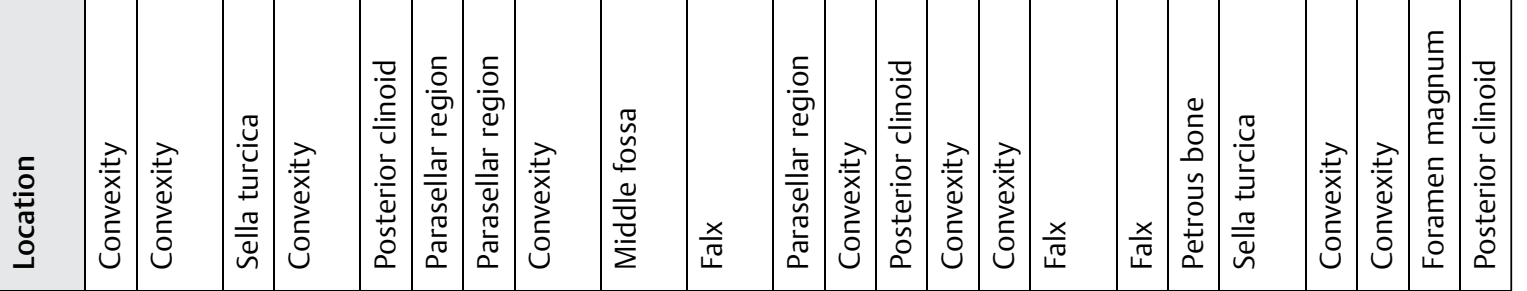

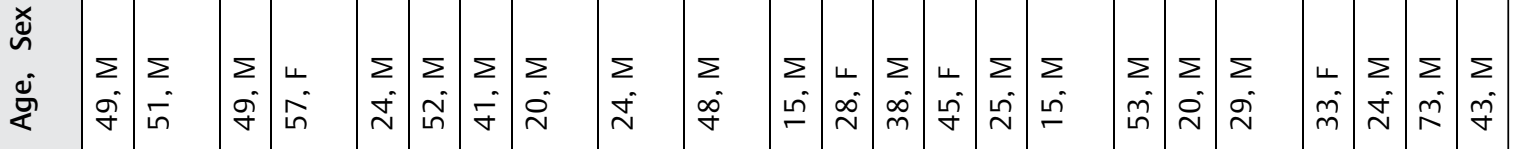
药

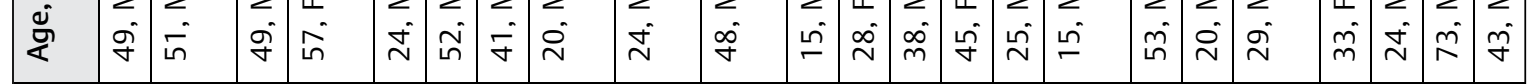

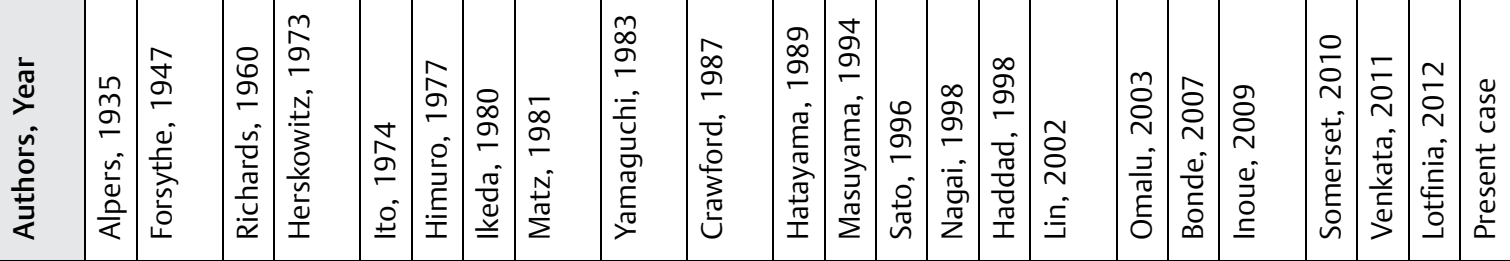

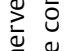

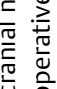
ż خ 은

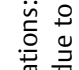
至营 


\section{Conclusion}

Intracranial osteochondromas are rare but should be included in the differential diagnosis of intracranial extra-axial tumors demonstrating atypical patterns of calcification. Thallium201 SPECT might be useful in differentiating osteochondromas from other intracranial tumors. Although the long-term treatment outcome of intracranial osteochondromas is still unknown because of the scarcity of previous data, the risk of the goal of total resection should be carefully weighed against the chance of recurrence given the benign nature of the disease.

\section{References}

1 Somerset HL, Kleinschmidt-DeMasters BK, Rubinstein D, Breeze $\mathrm{RE}$. Osteochondroma of the convexity: pathologic-neuroimaging correlates of a lesion that mimics high-grade meningioma. J Neurooncol 2010;98(3):421-426

2 Murphey MD, Choi JJ, Kransdorf MJ, Flemming DJ, Gannon FH. Imaging of osteochondroma: variants and complications with radiologic-pathologic correlation. Radiographics 2000;20(5): 1407-1434

3 Beck DW, Dyste GN. Intracranial osteochondroma: MR and CT appearance. AJNR Am J Neuroradiol 1989;10 (5, Suppl):S7-S8

4 Bonde V, Srikant B, Goel A. Osteochondroma of basi-occiput. Neurol India 2007;55(2):182-183

5 Crawford SC, Cornell SH, Franken EA. General case of the day. Intracranial osteochondroma. Radiographics 1987;7(6):1178-1180

6 Forsythe RW, Baker GS, et al. Intracranial osteochondroma. Proc Staff Meet Mayo Clin 1947;22(17):350-356

7 Haddad GF, Haddad FS, Zaatari G. Dural osteochondroma: case report, review of the literature and proposal of a new classification. Br J Neurosurg 1998;12(4):380-384

8 Hatayama T, Sekiya T, Suzuki S, Iwabuchi T. A case of intracranial osteochondroma: its MR images [in Japanese]. No Shinkei Geka 1989;17(11):1063-1066

9 Herskowitz A, el-Gammal T. Supratentorial cartilaginous tumors. (A report of 2 cases). Dis Nerv Syst 1973;34(7):384-388

10 Himuro H, Suzuki H, Takeyama E, Jinbo M, Kitamura K. A case of intracranial osteochondroma [in Japanese]. No Shinkei Geka 1977; 5(10):1079-1083

11 Ikeda Y, Shimura T, Higuchi H, Nakazawa S, Sugisaki Y. Intracranial osteochondroma-case report [in Japanese]. No Shinkei Geka 1980;8(3):263-269
12 Inoue $\mathrm{T}$, Takahashi $\mathrm{N}$, Murakami $\mathrm{K}$, Nishimura $\mathrm{S}$, Kaimori $\mathrm{M}$, Nishijima M. Osteochondroma of the sella turcica presenting with intratumoral hemorrhage. Neurol Med Chir (Tokyo) 2009; 49(1):37-41

13 Ito $\mathrm{U}$, Hashimoto $\mathrm{K}$, Inaba Y. Osteochondroma of the posterior clinoid process. Report of a case with special reference to its histogenesis. Acta Neuropathol 1974;27(4):329-335

14 Lin WC, Lirng JF, Ho DMT, et al. A rare giant intracranial osteochondroma. Zhonghua Yi Xue Za Zhi (Taipei) 2002;65(5):235-238

15 Lotfinia I, Vahedi P, Tubbs RS, Gavame M, Vahedi A. Basioccipital bone osteochondroma growing into the foramen magnum. Surg Neurol Int 2012;3:21

16 Mashiyama S, Sakurai Y, Kayama T, Nishino A, Jokura H. A case of intracranial osteochondroma originating with psychomotor epilepsy [in Japanese]. No Shinkei Geka 1994;22(2):165-168

17 Matz S, Israeli Y, Shalit MN, Cohen ML. Computed tomography in intracranial supratentorial osteochondroma. J Comput Assist Tomogr 1981;5(1):109-115

18 Nagai S, Yamamoto N, Wakabayashi K, et al. Osteochondroma arising from the convexity dura mater. Case illustration. J Neurosurg 1998;88(3):610

19 Omalu BI, Wiley CA, Hamilton RL. February 2003: a 53-year-old male with new onset seizures. Brain Pathol 2003;13(3):419-420, 423

20 Richards WW, Thompson MC. Suprasellar osteochondroma with chiasmal syndrome. Arch Ophthalmol 1961;65:437-441

21 Sato K, Kodera T, Kitai R, Kubota T. Osteochondroma of the skull base: MRI and histological correlation. Neuroradiology 1996; 38(1):41-43

22 Venkata RI, Kakarala SV, Garikaparthi S, Duttaluru SS, Parvatala A, Chinnam A. Giant intracranial osteochondroma: a case report and review of the literature. Surg Neurol Int 2011;2:118

23 Yamaguchi T, Wada S, Matsukado Y. Intracranial giant osteochondroma with unusual clinical features [in Japanese]. No Shinkei Geka 1983;11(2):181-184

24 Bakdash H, Alksne JF, Rand RW. Osteochondroma of the base of the skull causing an isolated oculomotor nerve paralysis. Case report emphasizing microsurgical techniques. J Neurosurg 1969;31(2): 230-233

25 Fon G, Sage MR. Osteochondroma of the clivus. Australas Radiol 1979;23(1):46-53

26 Alpers BJ. Cerebral osteochondroma of dural origin. Ann Surg 1935;101(1):27-37

27 Bovée JV, Hogendoorn PCW. Multiple osteochondromas. In World Health Organization classification of tumours. Pathology and genetics of tumours of soft tissue and bone. Edited by: Fletcher CDM, Unni KK and Mertens F. Lyon, IARC Press; 2002: 360-362 